\title{
ISOMORPHISM TYPES OF INFINITE SYMMETRIC GRAPHS
}

\author{
C. $M$. $B A N G^{1}$
}

\begin{abstract}
Professor Bjarni Jónsson asked about the cardinality of isomorphism types of infinite symmetric graphs of order $m$, for each infinite cardinal $m$. We show that there are $2^{m}$ pairwise nonisomorphic infinite symmetric graphs of order $m$, for each infinite cardinal $m$.
\end{abstract}

A symmetric graph is an ordered pair $\langle U, F\rangle$ where $F$ is a symmetric relation over the set $U$. The cardinality of $U$ is referred to as the order of the graph. Professor Bjarni Jónsson stated in [2, p. 31] that, as far as we know, the cardinality of the class of all pairwise nonisomorphic infinite symmetric graphs of order $m$, for each infinite cardinal $m$, is unknown. Since $F$ is a subset of $U \times U$, it is trivial to see that $2^{m}$ is an upper bound. In this paper, we shall settle this cardinality question by proving the following theorem.

THEOREM. The cardinality of the isomorphism types of infinite symmetric graphs of order $m$ is $2^{m}$ for each infinite cardinal $m$.

We base our proof on the result by Professors Comer and LeTourneau [1] that there are $2^{m}$ pairwise nonisomorphic 1-unary root algebras of order $m$, for each infinite cardinal $m$. With each 1-unary root algebra $A=\langle U, F\rangle$, we associate the symmetric graph $\bar{A}=\langle U, F\rangle$ where $F=f \cup f^{-1}$. To complete the proof of our theorem, it is therefore sufficient to prove the following lemma.

Lemma. Let $\bar{A}=\langle U, F\rangle$ and $\bar{B}=\langle U, G\rangle$ be two symmetric graphs associated with two 1-unary root algebras $A=\langle U, f\rangle$ and $B=\langle U, g\rangle$, respectively. If $\bar{A}$ and $\bar{B}$ are two isomorphic symmetric graphs, then $A$ and $B$ are two isomorphic 1-unary root algebras.

Let $a$ be the fixed point of $A=\langle U, f\rangle$. Note that $a$ is the only fixed point, i.e., it is the only element of $U$ satisfying $f(a)=a$. Let us write $x \rightarrow^{f} y$ or

Received by the editors November 15,1971 .

AMS 1970 subject classifications. Primary 05C30, 08 A05.

Key words and phrases. Isomorphism types, infinite symmetric graphs, 1-unary root algebra.

${ }^{1}$ The author wants to express his gratitude to the referee for his suggestions concerning the style of presentation. 
$y \leftarrow^{f} x$ if $f(x)=y$. (1) For each point $x \in U$, there is one and only one arrow leaving $x$. (2) The loop arrow $a \rightarrow^{f} a$ is the only arrow leaving the fixed point $a$. (3) For each $x(\neq a) \in U$ there is a least positive integer $n$, depending on $x$, satisfying $f^{n}(x)=a$. We shall call $n$ the height of $x$. Then, we have connecting arrows from $x$ to $a$ as follows:

$$
x \stackrel{f}{\longrightarrow} f(x) \stackrel{f}{\longrightarrow} \ldots \stackrel{f}{\longrightarrow} f^{n-1}(x) \stackrel{f}{\longrightarrow} f^{n}(x)=a
$$

with $f^{n-1}(x) \neq f^{n}(x)$, i.e., $f^{n-1}(x) \neq a$.

Proof. Assume that $\phi$ is a symmetric graph isomorphism from $\bar{A}=$ $\langle U, F\rangle$ onto $\bar{B}=\langle U, G\rangle$. We shall show that $\phi$ is a 1-unary root algebra isomorphism from $A=\langle U, f\rangle$ onto $B=\langle U, g\rangle$. Since $\phi$ already is a bijection on $U$, it suffices to show that $g(\phi(x))=\phi(f(x))$ for each $x \in U$, or equivalently,

$$
\phi(x) \stackrel{g}{\longrightarrow} \phi(f(x)) \text { for each } x \in U .
$$

In other words, it is sufficient to show that $\phi$ is arrow preserving.

Since $(x, f(x)) \in f \subseteq f \cup f^{-1}=F$ and since $\phi$ is a symmetric graph isomorphism from $\bar{A}=\langle U, F\rangle$ to $\bar{B}=\langle U, G\rangle$, we have $\phi(x, f(x)) \in G$. But, then

which means

$$
\left(\phi(x), \phi(f(x))=\phi(x, f(x)) \in G=g \cup g^{-1},\right.
$$

$$
\phi(x) \stackrel{g}{\stackrel{\text { or }}{\rightleftarrows}} \phi(f(x))
$$

in an obvious sense. Note that to prove $\left(^{*}\right)$ is to prove that the top arrow of (4) holds.

If $x=a$, then (4) coincides with $\phi(a) \rightarrow^{g} \phi(a)$ in either case which shows, first, that $\left(^{*}\right)$ is true in case $x=a$ and, second, that

$$
\phi(a) \text { is the fixed point, say, } b \text { of } \bar{B}=\langle U, G\rangle .
$$

If $x \neq a$, let $n(\geqq l)$ be the height of $x$. By our observations (3), (4) and (5), we have

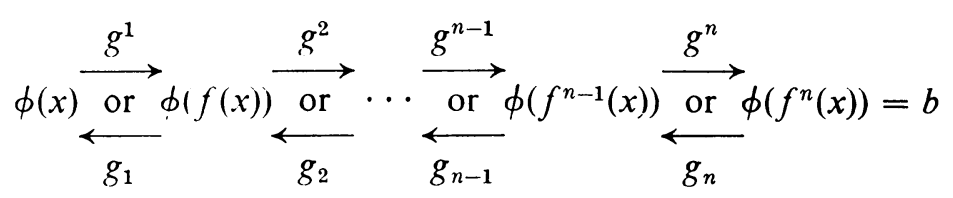

with $\phi\left(f^{n-1}(x)\right) \neq \phi\left(f^{n}(x)\right)=b$. (The upper and lower subscripts of $g$ are attached only for the convenience of ensuing quotations.) By our early 
observation (2) we see that $g^{n}$ holds while $g_{n}$ does not. Since $g^{n}$ already is an arrow leaving the element $\phi\left(f^{n-1}(x)\right)$ in $\bar{B}$, there can be no other arrow leaving $\phi\left(f^{n-1}(x)\right)$ by observation (1). Hence, $g^{n-1}$ must hold, while $g_{n-1}$ does not. Similarly applying (1) over and over, we shall have

$$
\phi(x) \stackrel{g}{\longrightarrow} \phi(f(x)) \stackrel{g}{\longrightarrow} \cdots \stackrel{g}{\longrightarrow} \phi\left(f^{n-1}(x)\right) \stackrel{g}{\longrightarrow} \phi\left(f^{n}(x)\right)=b
$$

the first arrow (from the left) of which surely proves $\left({ }^{*}\right)$. This completes a proof of our lemma and, consequently, our theorem.

Recall [1] that each of the aforementioned Comer-LeTourneau 1-unary root algebras has only the trivial automorphism group. From this, the following is immediate.

COROLlaRY. There are $2^{m}$ pairwise nonisomorphic infinite symmetric graphs of order $m$, for each infinite cardinal $m$, each with only the trivial automorphism group.

\section{REFERENCES}

1. S. D. Comer and J. J. LeTourneau, Isomorphism types of infinite algebras, Proc. Amer. Math. Soc. 21 (1969), 635-639. MR 39 \#116.

2. B. Jónsson, Topics in universal algebra, Lecture notes, Vanderbilt University, 1971, 212 pp.

Department of Mathematics, Emory University, Atlanta, Georgia 30322 